\title{
Long-term administration of escitalopram in patients with social anxiety disorder in Japan
}

This article was published in the following Dove Press journal:

Neuropsychiatric Disease and Treatment

22 July 2016

Number of times this article has been viewed

\author{
Satoshi Asakura' \\ Taiji Hayano² \\ Atsushi Hagino ${ }^{2}$ \\ Tsukasa Koyama ${ }^{3}$ \\ 'Health Care Center and Department \\ of Psychiatry, Hokkaido University \\ Graduate School of Medicine, Sapporo, \\ Hokkaido, ${ }^{2}$ Clinical Development \\ Planning and Management, Mochida \\ Pharmaceutical Co., Ltd, Tokyo, \\ ${ }^{3}$ Clinical Research Center, Oyachi \\ Hospital, Sapporo, Hokkaido, Japan
}

Correspondence: Satoshi Asakura Health Care Center and Department of Psychiatry, Hokkaido University Graduate School of Medicine, North 16, West 7, Sapporo, Hokkaido 060-08I6, Japan Tel +8I II 70654 I8

Fax $+8 \mid$ | | 706508 |

Email asakurap@academic.hokudai.ac.jp
Purpose: To investigate the safety, tolerability, and effectiveness of escitalopram in patients with social anxiety disorder in Japan.

Methods: A 52-week, open-label study was conducted in Japanese patients with social anxiety disorder with a total score $\geq 60$ on the Liebowitz Social Anxiety Scale - Japanese Version (LSAS-J) and $\geq 4$ on the Clinical Global Impression - Severity Scale. Escitalopram $10 \mathrm{mg}$ /day was administered for the first week and could be increased to $20 \mathrm{mg} / \mathrm{day}$.

Results: The study included 158 patients: $81.0 \%$ (128/158) completed 52 weeks of escitalopram treatment, $68.4 \%(108 / 158)$ increased their dose to $20 \mathrm{mg} /$ day, and $56.3 \%(89 / 158)$ remained on $20 \mathrm{mg} /$ day. Adverse drug reactions were reported by $57.6 \%(91 / 158)$ of patients. The most common (incidence $\geq 10 \%$ ) were somnolence and nausea. The incidence of adverse drug reactions was similar in extensive and poor metabolizers of cytochrome P450 2C19. No adverse drug reactions increased in incidence by $>5 \%$ after week 12 . The incidence of serious adverse events was $1.3 \%(2 / 158)$. No deaths occurred. The LSAS-J total scores improved until week 52. The LSAS-J response rate ( $\geq 30 \%$ improvement in LSAS-J) was $69.0 \%$, the Clinical Global Impression - Improvement Scale response rate $(\leq 2)$ was $73.0 \%$, and the LSAS-J remission rate $(\leq 30)$ was $27.0 \%$.

Conclusion: In this first 52-week clinical study of social anxiety disorder, escitalopram $10-20 \mathrm{mg} /$ day was safe, well tolerated, and effective in Japanese patients.

Keywords: escitalopram, Japanese, long-term study, social anxiety disorder, selective serotonin reuptake inhibitors

\section{Introduction}

Social anxiety disorder (SAD) is a psychiatric disorder characterized by considerable fear of social situations or activities, in which the patient could become the focus of attention of others, and by avoiding of anxiety-provoking situations. ${ }^{1} \mathrm{SAD}$ is generally considered a chronic disorder. ${ }^{2}$ Because patients with SAD tend to become socially isolated through continued avoidance of social situations, SAD affects their educational, professional, and economic status ${ }^{3}$ resulting in considerable loss not only to the patients themselves and their families, but also to society overall. Additionally, anxiety disorders, including SAD, are risk factors for suicidal ideation and suicide attempts and these risks are increased further by concurrent mood or anxiety disorders. ${ }^{4}$

In Japan, taijin-kyofu (TK) is a syndrome similar to SAD characterized by interpersonal sensitivity and fear and avoidance of interpersonal situations. The Diagnostic and Statistical Manual of Mental Disorders-5 emphasized in the "CultureRelated Diagnostic Issues" section on SAD that "The syndrome of taijin kyofusho (eg, in Japan and Korea) is often characterized by social-evaluative concerns, fulfilling the criteria for social anxiety disorder." TK has two subtypes - the "sensitive" 
and the "convinced" (c-TK; also called the "offensive" subtype). ${ }^{5}$ The common characteristics of c-TK are: 1) patients firmly believe that they have serious shortcomings, such as unpleasant body odor, inadequate eye expression, perceived physical defects, or stiff facial expression; 2) patients intuit from the behavior and actions of others that these shortcomings exist; and 3) patients perceive that these defects make others feel unpleasant and therefore must be corrected or removed by all means. ${ }^{6}$

Treatments for SAD include pharmacotherapy and psychotherapy, which may be used alone or in combination. ${ }^{7}$ Selective serotonin reuptake inhibitors or serotonin/ noradrenaline reuptake inhibitors are considered the first-line pharmacotherapy, and their efficacy has been demonstrated in double-blind studies. ${ }^{7-12}$ A long-term perspective is needed when treating SAD, and if a drug recommended by the treatment guidelines and/or treatment algorithms proves effective, treatment should be continued for 12 months in patients responsive to treatment. ${ }^{8,9,11}$

Escitalopram (ESC) is metabolized primarily by cytochrome P450 (CYP) 2C19, and patients are classified by CYP2C19 polymorphisms into either extensive metabolizers (EMs) or poor metabolizers (PMs). The proportion of PMs varies with ethnicity, being $2 \%-5 \%$ in Caucasians, but $15 \%-23 \%$ in Asians, including Japanese. ${ }^{13}$ In a clinical pharmacokinetic study conducted in Japan, the $\mathrm{AUC}_{0-\infty}$ of ESC in plasma in CYP2C19 EM subjects was about twice that in PM subjects. ${ }^{14}$

In this study, ESC was administered to patients with SAD in Japan for 52 weeks using flexible doses of 10-20 mg/day to investigate the safety and efficacy of long-term use of ESC, focusing on stratified analysis by CYP2C19 genotype.

\section{Methods}

\section{Study design}

This multicenter, open-label, flexible-dose, long-term study was conducted in 50 centers in Japan from December 2012 to October 2014. All centers obtained approval from their respective institutional review boards before initiating study treatment. This study complied with the Declaration of Helsinki and the Japanese Ministerial Ordinance on Good Clinical Practice for Drugs. Investigators obtained written informed consent from all patients who participated in this study. Patients' anonymity was preserved.

\section{Study patients}

Patients with SAD as their primary diagnosis according to the Diagnostic and Statistical Manual of Mental Disorders, 4th Edition - Text Revision ${ }^{15}$ were eligible for this study.
Patients were diagnosed using the Mini-International Neuropsychiatric Interview Japanese version 5.0.0. ${ }^{16}$ Eligible patients of either sex were aged $\geq 18$ and $\leq 64$ years. Additionally, patients were required to have a total score $\geq 60$ on the Liebowitz Social Anxiety Scale - Japanese Version (LSAS-J) ${ }^{17}$ and $\geq 4$ on the Clinical Global Impression - Severity Scale (CGI-S) and to exhibit fear/anxiety or avoidance traits in at least four items of the LSAS-J, of which $\geq 2$ were social interaction items at screening and baseline visits. Patients with a total score $\geq 15$ on the Montgomery Åsberg Depression Rating Scale ${ }^{18}$ were excluded from the study.

\section{Dosing method}

The study comprised a 1-week screening period, 52 weeks of treatment, and a 2-week follow-up period. ESC $10 \mathrm{mg}$ tablets were administered orally once daily during the treatment period. After the first week of this study, during which patients received $10 \mathrm{mg} /$ day of ESC, the dosage was flexible (10 or $20 \mathrm{mg} /$ day) and could be increased, maintained, or decreased at the discretion of the investigator.

\section{Endpoints and evaluation method}

The following six indicators were used for safety evaluation: adverse events (AEs); clinical laboratory values (hematology, biochemistry, and urinalysis); vital signs (systolic blood pressure, diastolic blood pressure, and pulse rate); body weight; standard 12-lead electrocardiogram (ECG); and the Columbia Suicide Severity Rating Scale. ${ }^{19}$ Clinical laboratory values and standard 12-lead ECG parameters were measured by the central laboratory.

For efficacy evaluation, the LSAS-J and the Japanese version of the Sheehan Disability Scale (SDISS) ${ }^{20}$ were assessed at baseline visits, at weeks 2, 4, 8, 12, 16, 20, 24, 32,40 , and 52 or at discontinuation of treatment. The CGI was assessed at baseline visits, at weeks 2, 4, 8, 12, 16, 20, $24,28,32,36,40,44,48$, and 52 , or at discontinuation of treatment. Social Anxiety/Taijin-Kyofu Scale (SATS) ${ }^{5}$ was assessed at baseline visits, at weeks 12, 24, and 52, or at discontinuation of treatment.

Of these, the LSAS-J, CGI-S, and Clinical Global Impression - Improvement Scale (CGI-I) are commonly used in clinical studies targeting patients with SAD. The LSAS is an outcome measure for SAD that comprises two subscales, fear/anxiety and avoidance, each consisting of 24 items. The Japanese version, the LSAS-J, is used in Japan. The SATS is a structured interview-style outcome measure comprising three subscales: fear/anxiety, avoidance behaviors, and c-TK's cognitive symptoms (thinking that one's body odor, eye expression, physical appearance, or facial expression 
makes other people feel unpleasant and knowing, based on how others behave, that these shortcomings exist), each consisting of four items (each item ranges from 0 to 4 , with a maximum total of 16 per subscale, for a total maximum of 48), which are used to evaluate both SAD and TK. SDISS is a patient-reported evaluation of functional changes in daily life. Raters received training in advance on the LSAS-J and SATS to standardize assessments.

In this study, the CYP2C19 genotype for each patient was determined, and safety and effectiveness were stratified by CYP2C19 genotype.

\section{Analysis}

Analysis sets

The safety analysis set comprised patients administered ESC at least once. The efficacy analysis set comprised patients in the safety analysis set with at least one valid postbaseline LSAS-J assessment (full analysis set [FAS]).

\section{Analytical methods}

For safety, summary statistics were calculated for AEs and adverse drug reactions (ADRs) $(\mathrm{ADRs}=\mathrm{AEs}$ where a causal relationship to the investigational drug could not be ruled out) incidences, clinical laboratory test values, vital signs, body weight, ECG, and Columbia Suicide Severity Rating Scale. The Medical Dictionary for Regulatory Activities Ver 16.0 was used for AE preferred terms. For efficacy, summary statistics were calculated for remission rates based on LSAS-J total scores (percentage of patients with LSAS-J total score $\leq 30$ ), response rates based on LSAS-J total scores (percentage of patients with a decrease $\geq 30 \%$ from baseline in LSAS-J total score) and CGI-I (CGI-I $\leq 2)$, and changes in the LSAS-J total score, SATS total score, CGI-S and SDISS total score, and CGI-I.

\section{Results \\ Patient background}

Figure 1 shows the breakdown of patients included in this study. All 158 patients were included in both efficacy and safety analysis sets. The 52-week treatment period was completed by $81.0 \%(128 / 158)$ of patients. The overall withdrawal rate was $19.0 \%(30 / 158)$. AEs $(10.8 \%, 17 / 158)$ were the most common reason for withdrawal, followed by withdrawal of consent $(8.2 \%, \mathrm{n}=13)$.

Table 1 shows demographic and other baseline characteristics of the study population. There was an equal distribution of males and females. Age (mean \pm SD) was $33.3 \pm 10.8$ years. The percentage of patients who were CYP2C19 PMs was $13.3 \%(21 / 158)$.

All patients received $10 \mathrm{mg} /$ day ESC for the first week and $31.6 \%(n=50)$ stayed on $10 \mathrm{mg} /$ day throughout the entire treatment period. Of the 108 patients $(68.4 \%)$ who increased their dose to $20 \mathrm{mg} /$ day, 89 patients $(56.3 \%)$ stayed on this dose. The final daily dose of ESC was $10 \mathrm{mg}$ for 66 patients (41.8\%) and $20 \mathrm{mg}$ for 92 patients (58.2\%). Over $83.5 \%$ of patients $(n=132)$ were exposed to ESC for more than 36 weeks. Participating patients were exposed to ESC for $311 \pm 111$ days (mean \pm SD). Treatment compliance, as measured by pill counts, was $\geq 75 \%$ for all but one patient.

\section{Safety}

\section{Adverse events}

The incidence of AEs was 82.9\% (131/158). Median time to onset of AEs with an incidence of $\geq 2 \%$ was 5.5 days and

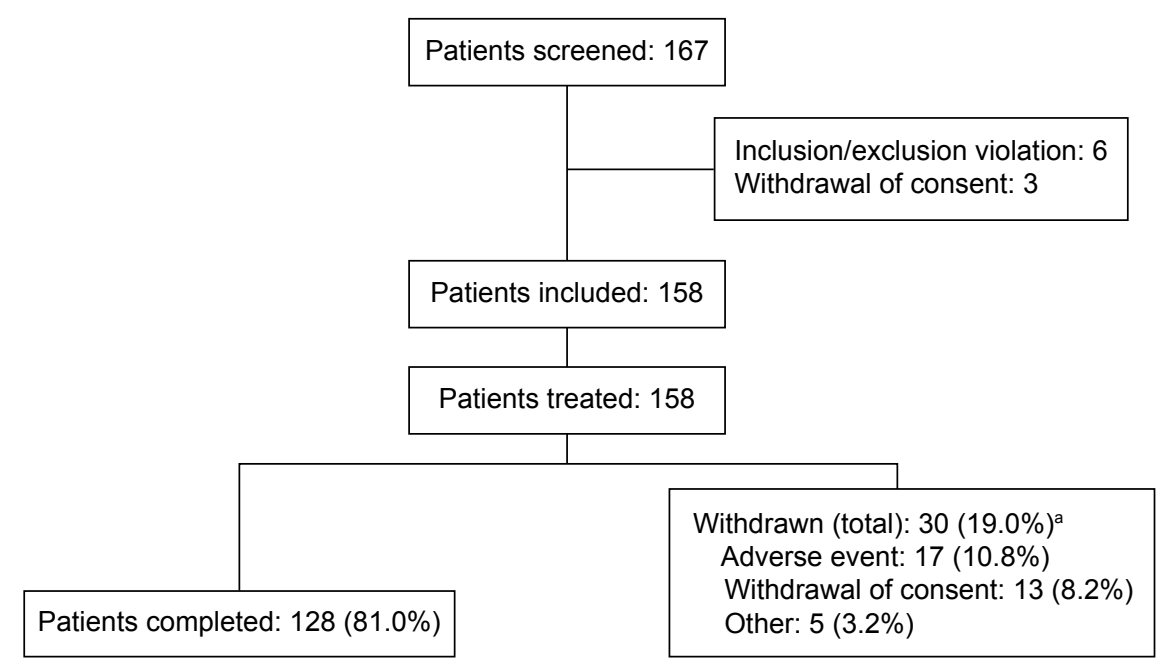

Figure I Flow chart of patient disposition.

Note: ${ }^{a}$ Multiple answers may be given for reason for withdrawal. 
Table I Demographic and other baseline characteristics (APTS, $\mathrm{n}=158)$

\begin{tabular}{|c|c|c|}
\hline Item & Category & Total (I 58 patients) \\
\hline \multicolumn{3}{|l|}{ Sex } \\
\hline \multirow[t]{2}{*}{ Number of patients (\%) } & Male & $79(50.0)$ \\
\hline & Female & $79(50.0)$ \\
\hline \multicolumn{3}{|l|}{ Age (years) } \\
\hline Mean \pm SD & & $33.3 \pm 10.8$ \\
\hline \multicolumn{3}{|l|}{ Body weight (kg) } \\
\hline Mean \pm SD & & $60.1 \pm 13.0$ \\
\hline \multicolumn{3}{|l|}{ BMI $\left(\mathrm{kg} / \mathrm{m}^{2}\right)$} \\
\hline Mean \pm SD & & $22.2 \pm 4.0$ \\
\hline \multicolumn{3}{|l|}{ CYP2C19 genotype } \\
\hline \multirow[t]{2}{*}{ Number of patients (\%) } & $\mathrm{EM}$ & I 37 (86.7) \\
\hline & PM & $21(13.3)$ \\
\hline \multicolumn{3}{|l|}{ Age at SAD onset (years) } \\
\hline Mean \pm SD & & $19.0 \pm 9.7$ \\
\hline \multicolumn{3}{|l|}{ Duration of SAD (years) } \\
\hline Mean \pm SD & & $14.3 \pm 12.0$ \\
\hline \multicolumn{3}{|l|}{ Total LSAS-J score } \\
\hline Mean \pm SD & & $95.3 \pm 19.5$ \\
\hline Median & & 94.0 \\
\hline Minimum-maximum & & $60-144$ \\
\hline \multicolumn{3}{|l|}{ Total SATS score } \\
\hline Mean \pm SD & & $23.7 \pm 6.9$ \\
\hline Median & & 22.5 \\
\hline Minimum-maximum & & $11-42$ \\
\hline \multicolumn{3}{|l|}{ CGI-S } \\
\hline Mean \pm SD & & $4.9 \pm 0.9$ \\
\hline Median & & 5.0 \\
\hline Minimum-maximum & & $4-7$ \\
\hline \multicolumn{3}{|l|}{ Total SDISS score } \\
\hline Mean \pm SD & & $1 I .1 \pm 6.6$ \\
\hline Median & & 11.0 \\
\hline Minimum-maximum & & $0-30$ \\
\hline \multicolumn{3}{|l|}{ Total MADRS score } \\
\hline Mean \pm SD & & $4.0 \pm 4.0$ \\
\hline Median & & 3.0 \\
\hline Minimum-maximum & & $0-14$ \\
\hline
\end{tabular}

Abbreviations: APTS, all-patients-treated set; BMI, body mass index; CGI-S, Clinical Global Impression - Severity Scale; CYP, cytochrome P450; EM, extensive metabolizer; LSAS-J, Liebowitz Social Anxiety Scale - Japanese Version; MADRS, Montgomery Åsberg Depression Rating Scale; PM, poor metabolizer; SAD, social anxiety disorder; SATS, Social Anxiety/Taijin-Kyofu Scale; SD, standard deviation; SDISS, the Japanese version of Sheehan Disability Scale.

median duration was 11 days, that is, occurring within the first 1-2 weeks and transient.

The incidence of ADRs was 57.6\% (91/158). All ADRs were either mild or moderate in severity; Table 2 shows the ADRs with an incidence $\geq 2 \%$. The most common (incidence $\geq 10 \%$ ) were somnolence and nausea. By time of onset (12-week intervals), the incidence of ADRs was highest in the period up until the end of week $12(52.5 \%)$ and decreased subsequently from $10.6 \%$ to $4.5 \%$ from week 13 through 48 . In all periods after week 13 , the incidence of individual ADRs did not increase by $\geq 5 \%$ after week 12 . The proportion of

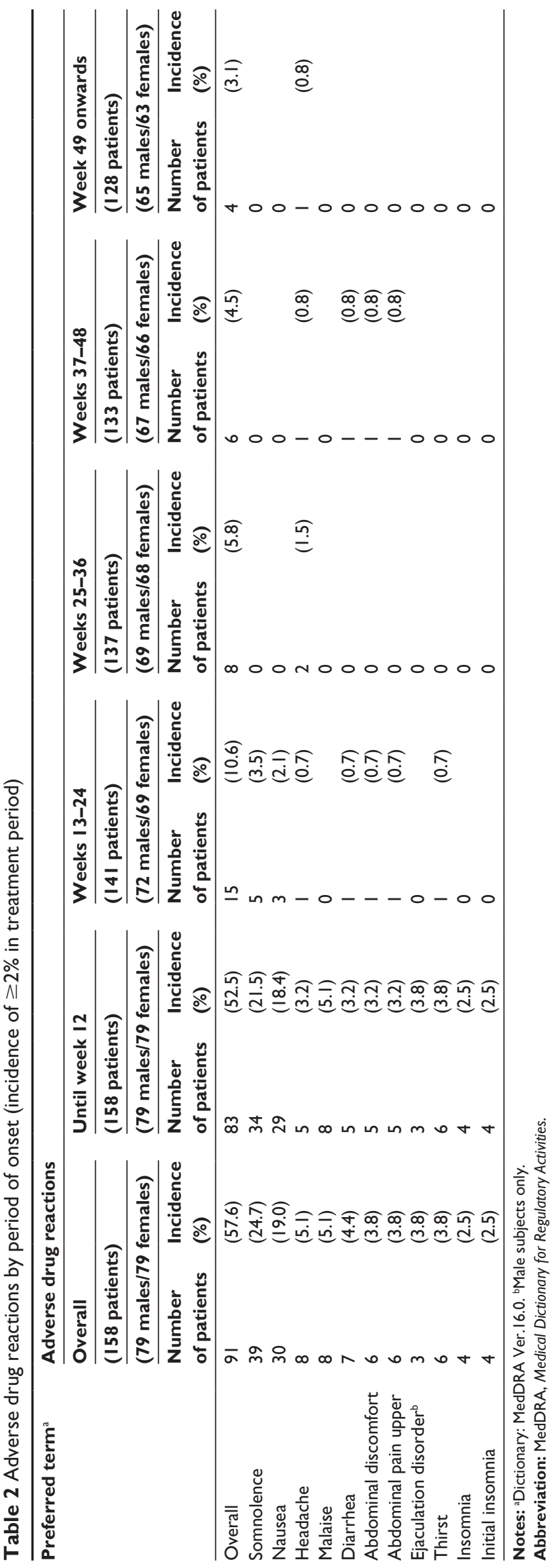


patients reporting ADRs by CYP2C19 genotype was 58.4\% $(80 / 137)$ in EMs and 52.4\% (11/21) in PMs.

\section{Serious AEs and AEs leading to discontinuation}

Two patients reported serious AEs (1.3\%), both during the treatment period: deep vein thrombosis and anal fissure. Deep vein thrombosis was the only severe event that occurred in this study. Neither of these events was considered by the investigator to be treatment-related. No deaths occurred in this study. No women became pregnant during this study. In this study, the incidence of AEs leading to discontinuation of treatment was $11.4 \%(18 / 158)$, most of which $(13 / 18)$ occurred by week 12 .

\section{Other evaluation indicators}

Based on the Columbia Suicide Severity Rating Scale, suicidal behavior was not detected in any patients at the start of treatment. Suicidal ideation was exacerbated from the start of treatment in $6.3 \%(10 / 158)$ patients; among them, seven patients were not judged to have suicide-related AEs, and three patients (five cases) were judged to have either mild or moderate AEs ("suicidal ideation") and eventually recovered. Of these five events, one was considered treatment-related by the investigator.

There were no clinically significant safety findings regarding changes in mean values of vital signs or clinical laboratory values. All mean clinical safety laboratory values were within reference ranges.

The weight increase (mean \pm standard deviation [SD]) from baseline to last assessment was $1.9 \pm 3.6 \mathrm{~kg}(\mathrm{n}=158)$. Weight increase was reported as an $\mathrm{AE}$ by three patients (1.9\%, three cases). All three AEs were considered treatmentrelated by the investigator. One patient withdrew due to weight increase. Weight and body mass index increased from $59.5 \mathrm{~kg}$ and $25.4 \mathrm{~kg} / \mathrm{m}^{2}$ at baseline to $64.2 \mathrm{~kg}$ and $27.4 \mathrm{~kg} / \mathrm{m}^{2}$ at withdrawal (219 days after the start of treatment).

There were no clinically significant changes in the mean values of ECG parameters. The changes (mean \pm SD) from baseline to last assessment of treatment in RR, PR, QRS,

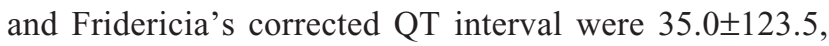
$0.1 \pm 12.7,2.1 \pm 6.4$, and $5.4 \pm 15.2 \mathrm{~ms}$, respectively. The change (mean \pm SD) from baseline to last assessment of treatment in the Fridericia's corrected QT interval was $2.4 \pm 12.0 \mathrm{~ms}$ $(\mathrm{n}=67)$ for the $10 \mathrm{mg}$ dose and $7.7 \pm 16.9 \mathrm{~ms}(\mathrm{n}=91)$ for the $20 \mathrm{mg}$ dose. No patients had a QTcF interval $>500 \mathrm{~ms}$ during treatment. Ten patients $(6.3 \%)$ had a change from baseline to last assessment $>30 \mathrm{~ms}$ and one patient had a change $>60 \mathrm{~ms}$ (68 $\mathrm{ms}$ at week 52 and a QTcF of $469 \mathrm{~ms}$ ).
By CYP2C19 genotype, the changes (mean \pm SD) from baseline to end of treatment in the QTcF interval were 5.7 $\pm 15.1 \mathrm{~ms}(\mathrm{n}=137)$ in EMs and 4.0 $\pm 16.3 \mathrm{~ms}(\mathrm{n}=21)$ in PMs.

\section{Effectiveness}

Table 3 shows the efficacy outcomes.

\section{LSAS-J}

The LSAS-J total score (mean \pm SD) decreased from $95.3 \pm 19.5$ at baseline to $49.9 \pm 28.0$ (observed cases [OC], FAS) at week 52 and $56.3 \pm 30.8$ at last assessment (last observation carried forward, FAS).

Figure 2 shows the change in LSAS-J total score (mean $\pm \mathrm{SD}$ ) by visit. The change in LSAS-J total score (mean $\pm \mathrm{SD}$ ) from baseline was $-26.6 \pm 21.5$ at week 12 $(n=141),-35.6 \pm 27.2$ at week $24(n=138)$, and $-44.8 \pm 28.8$ at week $52(\mathrm{n}=126)$ (OC, FAS).

Response rates based on $\geq 30 \%$ improvement in LSAS-J total score were $39.0 \%(55 / 141)$ at week $12,55.8 \%(77 / 138)$ at week 24, and 69.0\% (87/126) at week 52 (OC, FAS).

Remission rates based on LSAS-J total score $\leq 30$ were $5.0 \%(7 / 141)$ at week $12 ; 18.1 \%(25 / 138)$ at week 24 , and $27.0 \%(34 / 126)$ at week 52 (OC, FAS).

By CYP2C19 genotype, completion rates were $82.5 \%$ $(113 / 137)$ in EMs versus $71.4 \%$ (15/21) in PMs. Response rates were 69.4\% (77/111) in EMs and 66.7\% (10/15) in PMs (OC, FAS). Remission rates at week 52, based on LSAS-J total score, were $27.9 \%(31 / 111)$ in EMs and 20.0\% (3/15) in PMs (OC, FAS). Changes in LSAS-J total score (mean \pm SD) were $-45.1 \pm 28.5(n=111)$ in EMs and $-42.5 \pm 31.9(n=15)$ in PMs (OC, FAS).

\section{SATS}

Change in SATS total score (mean \pm SD) from baseline was $-5.4 \pm 5.7$ at week $12(n=141),-7.7 \pm 6.7$ at week $24(\mathrm{n}=138)$, and $-11.2 \pm 7.6$ at week $52(\mathrm{n}=126)(\mathrm{OC}, \mathrm{FAS})$. Of 158 patients, $66(41.8 \%)$ had SATS cognitive symptoms. Their disposition was as follows: 15 with "unpleasant body odor", ten with "inadequate eye expression", ten with "stiff facial expression", four with "perceived physical defects", and eight with "other;" 19 patients had multiple symptoms. For patients with c-TK's cognitive symptoms, changes in the SATS total score (mean $\pm \mathrm{SD}$ ) were $-5.6 \pm 6.5$ at week $12,-7.8 \pm 7.6$ at week 24 , and $-12.0 \pm 9.2$ at week 52 (Table 3). Additionally, 52 weeks of treatment with ESC resulted in a decrease in total scores in each of the fear/ anxiety, avoidance behavior, and cognitive symptom subscales. 
Table 3 Summary of efficacy evaluation (FAS)

\begin{tabular}{|c|c|c|c|c|}
\hline & $\begin{array}{l}\text { Baseline } \\
\text { (158 patients) }\end{array}$ & $\begin{array}{l}\text { Week } 12 \\
\text { (14I patients) }\end{array}$ & $\begin{array}{l}\text { Week } 24 \\
\text { (138 patients) }\end{array}$ & $\begin{array}{l}\text { Week } 52 \\
\text { (126 patients) }\end{array}$ \\
\hline LSAS-J total score & $95.3 \pm 19.5$ & $69.0 \pm 25.1$ & $59.9 \pm 28.7$ & $49.9 \pm 28.0$ \\
\hline Change & - & $-26.6 \pm 21.5$ & $-35.6 \pm 27.2$ & $-44.8 \pm 28.8$ \\
\hline SATS total score & $23.7 \pm 6.9$ & $18.3 \pm 8.0$ & $15.9 \pm 8.9$ & $12.5 \pm 8.7$ \\
\hline Change & - & $-5.4 \pm 5.7$ & $-7.7 \pm 6.7$ & $-11.2 \pm 7.6$ \\
\hline SATS total score (no cognitive symptoms) ${ }^{a}$ & $20.6 \pm 5.0$ & $15.6 \pm 6.8$ & $13.1 \pm 7.3$ & $10.0 \pm 6.6$ \\
\hline Change & - & $-5.3 \pm 5.0$ & $-7.7 \pm 6.2$ & $-10.7 \pm 6.2$ \\
\hline SATS total score (with cognitive symptoms) & $27.9 \pm 6.9$ & $22.1 \pm 8.0$ & $20.1 \pm 9.6$ & $16.0 \pm 10.1$ \\
\hline Change & - & $-5.6 \pm 6.5$ & $-7.8 \pm 7.6$ & $-12.0 \pm 9.2$ \\
\hline CGI-S & $4.9 \pm 0.9$ & $3.8 \pm 1.0$ & $3.3 \pm 1.1$ & $2.7 \pm 1.1$ \\
\hline Change & - & $-I . I \pm I . I$ & $-1.6 \pm 1.3$ & $-2.2 \pm 1.3$ \\
\hline CGI-I & - & $2.8 \pm 1.0$ & $2.4 \pm 1.0$ & $1.9 \pm 0.9$ \\
\hline SDISS (work/school) & $4.5 \pm 2.6$ & $3.1 \pm 2.4$ & $2.8 \pm 2.4$ & $2.2 \pm 2.2$ \\
\hline Change & - & $-1.3 \pm 2.3$ & $-1.6 \pm 2.6$ & $-2.2 \pm 2.6$ \\
\hline SDISS (social life) & $4.0 \pm 2.5$ & $2.8 \pm 2.1$ & $2.6 \pm 2.2$ & $2.0 \pm 2.0$ \\
\hline Change & - & $-1.1 \pm 2.1$ & $-1.3 \pm 2.4$ & $-1.9 \pm 2.4$ \\
\hline SDISS (communication and role at home) & $2.6 \pm 2.6$ & $1.9 \pm 2.1$ & $1.7 \pm 2.1$ & $1.6 \pm 1.9$ \\
\hline Change & - & $-0.6 \pm 1.9$ & $-0.8 \pm 2.3$ & $-0.9 \pm 2.2$ \\
\hline
\end{tabular}

Notes: Data presented as mean \pm SD. aBaseline: 92 patients, week 12: 83 patients, week 24: 82 patients, week $52: 74$ patients. ${ }^{\mathrm{b} B a s e l i n e: ~} 66$ patients, week I2: 58 patients, week 24: 56 patients, week 52: 52 patients.

Abbreviations: CGI-I, Clinical Global Impression - Improvement Scale; CGI-S, Clinical Global Impression - Severity Scale; FAS, full analysis set; LSAS-J, Liebowitz Social Anxiety Scale - Japanese Version; SATS, Social Anxiety/Taijin-Kyofu Scale; SD, standard deviation; SDISS, the Japanese version of Sheehan Disability Scale.

CGI

Analysis of CGI-S scores supported the clinical relevance of these long-term effectiveness results. CGI-S scores (mean $\pm \mathrm{SD}$ ) decreased throughout the study from $4.9 \pm 0.9$ at baseline to $2.7 \pm 1.1$ at week 52 (OC, FAS). Change in CGI-S (mean \pm SD) from baseline was $-1.1 \pm 1.1$ at week $12(\mathrm{n}=141),-1.6 \pm 1.3$ at week $24(\mathrm{n}=138)$, and $-2.2 \pm 1.3$ at week 52 ( $\mathrm{n}=126)$ (OC, FAS).

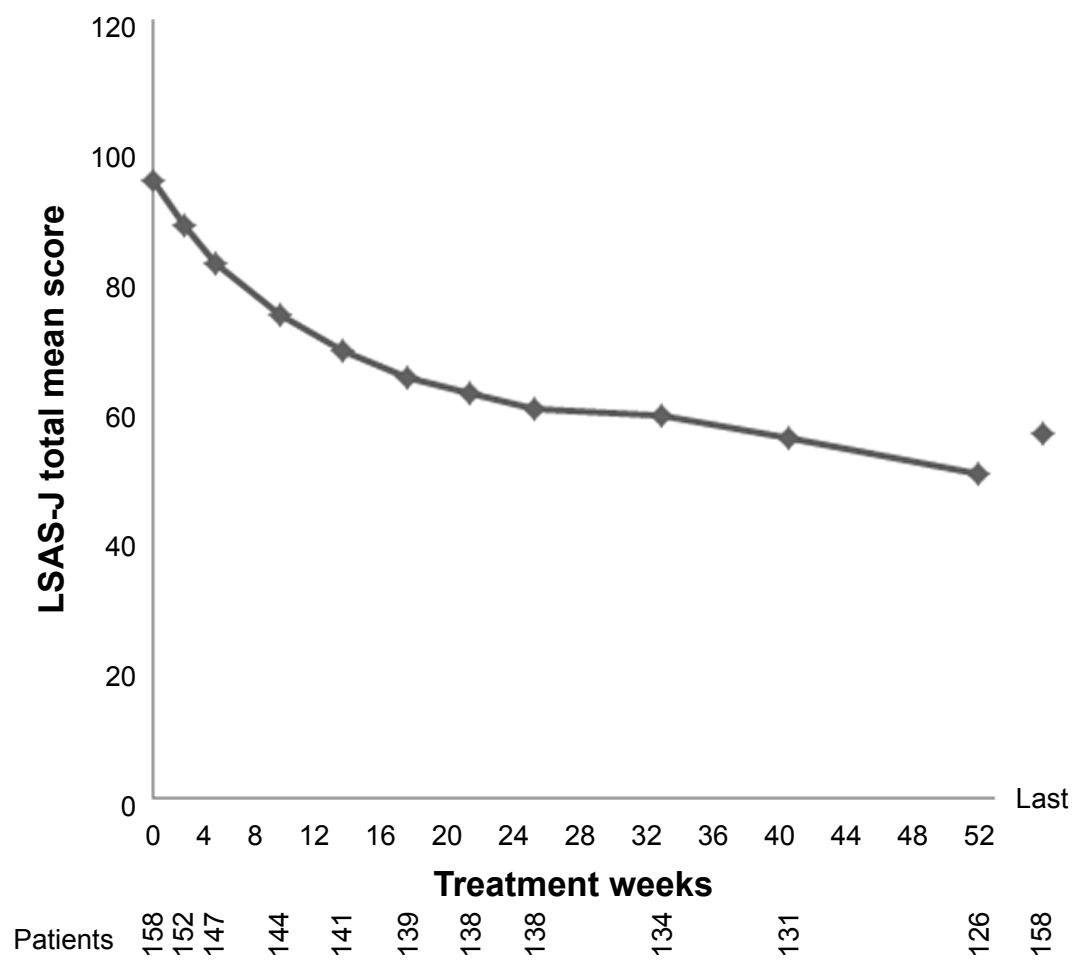

Figure 2 Estimated change in the Japanese version of the LSAS-J total scores from baseline to week 52 (FAS, OC by visit) and FAS, LOCF at last assessment. Note: Patient numbers at each visit are shown below the $x$-axis.

Abbreviations: FAS, full-analysis set; LOCF, last observation carried forward; LSAS-J, Liebowitz Social Anxiety Scale - Japanese Version; OC, observed cases. 
Patients who did not achieve sufficient efficacy with $10 \mathrm{mg} /$ day ESC (based on the CGI-S) had the dose increased to and maintained at $20 \mathrm{mg}$ /day ESC until the end of the treatment period. Patients were classified as improved $(\geq 1$ decrease in CGI-S), unchanged, or exacerbated ( $\geq 1$ increase in CGI-S). There were $78.7 \%$ (70/89) improved patients, $19.1 \%(17 / 89)$ unchanged patients, and 2.2\% (2/89) exacerbated patients.

CGI-I scores (mean \pm SD) decreased throughout the study from $3.6 \pm 0.6$ at week 2 to $1.9 \pm 0.9$ at week 52 (OC, FAS). Response rates based on the percentage of patients with CGI-I $\leq 2$ were $36.2 \%(51 / 141)$ at week $12 ; 52.2 \%$ (72/138) at week 24 ; and $73.0 \%(92 / 126)$ at week 52 (OC, FAS).

\section{SDISS}

Changes in SDISS (work/school) score (mean \pm SD) from baseline were $-1.3 \pm 2.3(\mathrm{n}=141)$ at week $12,-1.6 \pm 2.6$ $(\mathrm{n}=138)$ at week 24 , and $-2.2 \pm 2.6(\mathrm{n}=126)$ at week $52(\mathrm{OC}$, FAS). The corresponding changes in SDISS (social life) score (mean $\pm \mathrm{SD}$ ) from baseline were $-1.1 \pm 2.1,-1.3 \pm 2.4$, and $-1.9 \pm 2.4$, respectively (OC, FAS). The corresponding changes in SDISS (communication and role at home) score (mean $\pm \mathrm{SD}$ ) from baseline were $-0.6 \pm 1.9,-0.8 \pm 2.3$, and $-0.9 \pm 2.2$, respectively (OC, FAS).

\section{Montgomery Åsberg Depression Rating Scale}

The Montgomery Åsberg Depression Rating Scale total score (mean $\pm \mathrm{SD}$ ) improved from $4.0 \pm 4.0$ at baseline to $2.8 \pm 4.0$ (week 12), $2.4 \pm 3.3$ (week 24), and $2.1 \pm 4.2$ (week 52) (OC, FAS). Changes in Montgomery Åsberg Depression Rating Scale total score (mean $\pm \mathrm{SD}$ ) from baseline were $-1.0 \pm 3.3(\mathrm{n}=141)$ at week $12,-1.4 \pm 3.1(\mathrm{n}=138)$ at week 24 , and $-1.9 \pm 4.5(\mathrm{n}=126)$ at week 52 (OC, FAS).

\section{Discussion}

This is the first 52-week clinical study of ESC in SAD. The primary objective was to investigate the safety and tolerability of ESC in Japanese patients with SAD, and investigation of effectiveness was planned as a secondary objective. The completion rate of $81.0 \%$ was high for a study of this duration. The rate of withdrawal due to AEs in this study was $10.8 \%(17 / 158)$, similar to that reported in a long-term safety extension study with ESC (8.8\%) in patients with major depressive disorder in Europe/Canada. ${ }^{21}$

ADRs had an incidence of 57.6\% (91/158) and all were either mild or moderate; the most common were somnolence and nausea. There were no deaths and the incidence of serious AEs was $1.3 \%$ (2/158). No ADRs had an increase $>5 \%$ in incidence during the subsequent weeks of long-term ESC administration, compared with the period until week 12 . Most AEs were transient and occurred within the first week, with a median time of 5.5 days and a duration of 11 days. Most AEs leading to discontinuation of treatment occurred before week 12 , were either mild or moderate in severity, and eventually resolved. Suicidal ideation occurred in three patients; all these events were mild or moderate in severity, and all resolved.

ESC was safe and well tolerated in long-term treatment of Japanese patients with SAD with the majority of AEs being nonsevere and transient, with no new AEs that had not been seen during acute treatment.

In another randomized study comparing ESC and placebo in the treatment of patients with SAD for 24 weeks, the proportion of patients with AEs was $68.9 \%$ for $5 \mathrm{mg}$ /day ESC, $72.5 \%$ for $10 \mathrm{mg} /$ day ESC, and $78.2 \%$ for $20 \mathrm{mg}$ /day ESC; most of these AEs were either mild or moderate. ${ }^{12}$ These results were similar to those of the present study in Japan (74.7\% at week 24). Additionally, the most frequent AEs (reported by $\geq 10 \%$ of patients and more than twice that in the placebo group) occurring in the 10-20 mg groups were nausea, increased sweating, diarrhea, somnolence, and ejaculation disorder, ${ }^{12}$ and so no major differences were found between studies inside and outside Japan.

ESC is metabolized primarily by CYP2C19, and it is known that around $20 \%$ of Japanese are CYP2C19 PMs. The percentage of CYP2C19 PMs was $13.3 \%$ in this study. The $\mathrm{AUC}_{0-\infty}$ of ESC in plasma found in PM patients was about twice that in EM patients. Incidences of ADRs were similar in CYP2C19 EMs and PMs. No major differences were found between EMs and PMs in the effect on QTcF interval.

The mean weight gain was $1.9 \mathrm{~kg}$ from the start of the lead-in study and one patient withdrew from the study due to weight increase.

LSAS-J, the efficacy endpoint in this study, revealed an improving tendency over time by week 52 . The LSAS-J response rate ( $\geq 30 \%$ improvement in LSAS-J) was $69 \%$, CGI-I response rate (CGI-I $\leq 2)$ was $73 \%$, and LSAS-J remission rate (LSAS-J $\leq 30$ ) was $27 \%$ at week 52 . Trends for all SDISS subscores were similar, suggesting that efficacy was maintained, and quality of life may improve with long-term ESC administration. Furthermore, the $\geq 70 \%$ patients whose dose was increased and maintained at $20 \mathrm{mg} /$ day improved their CGI-S score, suggesting that increasing the ESC dose to $20 \mathrm{mg}$ /day in patients who did not improve on $10 \mathrm{mg}$ /day ESC may be useful. 
In this study, SATS was used as an efficacy endpoint to evaluate patients with SAD from a TK perspective. Patients believed to have c-TK (patients diagnosed with cognitive symptoms in SATS) comprised $41.8 \%$ of the study population (66/158). On each of the fear/anxiety, avoidance behavior, and c-TK's cognitive symptom subscales, use of ESC for 52 weeks resulted in decreased total scores, suggesting that administering ESC may also result in improved c-TK symptoms. Because the Diagnostic and Statistical Manual of Mental Disorders-5 notes that c-TK may also exist outside Asia, investigating the therapeutic efficacy of drugs in TK outside Japan may prove necessary in the future.

There are several notable limitations regarding this study. This study was an open-label, uncontrolled study conducted only in Japan, limiting the generalizability of findings. The study patients are a specific population defined by the inclusion and exclusion criteria.

In conclusion, this first 52-week clinical study showed that ESC 10-20 mg/day is safe, well tolerated, and effective in Japanese patients with SAD.

\section{Acknowledgments}

We thank the following investigators and their patients for participating in this study: Drs Yoshiko Kamekawa, Takashi Furune, Masashi Yoshida, Hiroshi Enguchi, Hiroaki Kusanagi, Toshiyuki Sakai, Katsuya Morishita, Tetsuo Abe, Yoko Ueda, Hiroaki Kaneko, Tsukasa Saito, Tadashi Aoki, Kunio Kato, Yuji Ikeuchi, Michihiro Takahashi, Shozo Chidori, Yasuaki Fujimaru, Masanobu Takeuchi, Ryunosuke Tsuruda, Kana Okamoto, Yuu Ariyoshi, Kouji Haramura, Kozaburou Oka, Ken Ikegami, Shigehiro Nakada, Yasumasa Asanobu, Akihiro Kakishima, Junichi Takeuchi, Naohiko Takiguchi, Akio Mantani, Kuninobu Yasuda, Wataru Miura, Michihiro Shimode, Hiroshi Nagao, Isao Fukunishi, Yasutaka Tawara, Seigen Gon, Naoyuki Hamada, Naoshige Matsuguchi, Junko Hatakeyama, Masahito Tsukamoto, Yuko Miura, Kimiyoshi Ozawa, Toshiaki Kuginuki, Taihei Fukuhara, Kahori Ito, Yoshinori Watanabe, Shigeru Itagaki, Hideji Uesugi, and Masahiko Fujita. This study was sponsored by Mochida Pharmaceutical Co, Ltd, where Taiji Hayano and Atsushi Hagino are employees. Drs Satoshi Asakura and Tsukasa Koyama served as medical experts for the study when Mochida Pharmaceutical Co, Ltd developed escitalopram to treat social anxiety disorder. Costs in preparing this paper were met by Mochida Pharmaceutical Co, Ltd.

\section{Author contributions}

All authors contributed toward data analysis, drafting and revising the paper and agree to be accountable for all aspects of the work.

\section{Disclosure}

The authors report no conflicts of interest in this work.

\section{References}

1. American Psychiatric Association. Diagnostic and Statistical Manual of Mental Disorders: DSM-5 ${ }^{\mathrm{TM}}$. 5th ed. Washington, DC: American Psychiatric Association; 2013.

2. Vriends N, Bolt OC, Kunz SM. Social anxiety disorder, a lifelong disorder? A review of the spontaneous remission and its predictors. Acta Psychiatr Scand. 2014;130(2):109-122.

3. Stein MB, Stein DJ. Social anxiety disorder. Lancet. 2008;371(9618): 1115-1125.

4. Sareen J, Cox BJ, Afifi TO, et al. Anxiety disorders and risk for suicidal ideation and suicide attempts: a population-based longitudinal study of adults. Arch Gen Psychiatry. 2005;62(11):1249-1257.

5. Asakura S, Inoue T, Kitagawa N, et al. Social Anxiety/Taijin-Kyofu Scale (SATS): development and psychometric evaluation of a new instrument. Psychopathology. 2012;45(2):96-101.

6. Yamashita I. Taijin-kyofu or Delusional Social Phobia. Sapporo: Hokkaido University Press; 1993.

7. Sareen J, Stein M. A review of the epidemiology and approaches to the treatment of social anxiety disorder. Drugs. 2000;59(3):497-509.

8. Swinson RP. Clinical practice guidelines. Management of anxiety disorders. Can J Psychiatry. 2006;51(2):35S-41S.

9. Bandelow B, Zohar J, Hollander E, et al. World Federation of Societies of Biological Psychiatry (WFSBP) guidelines for the pharmacological treatment of anxiety, obsessive-compulsive and post-traumatic stress disorders - first revision. World J Biol Psychiatry. 2008;9(4):248-312.

10. Baldwin DS, Anderson IM, Nutt DJ, et al. Evidence-based pharmacological treatment of anxiety disorders, post-traumatic stress disorder and obsessive-compulsive disorder: a revision of the 2005 guidelines from the British association for psychopharmacology. J Psychopharmacol. 2014;28(5):403-439.

11. Stein DJ, Baldwin DS, Bandelow B, et al. A 2010 evidence-based algorithm for the pharmacotherapy of social anxiety disorder. Curr Psychiatry Rep. 2010;12(5):471-477.

12. Lader M, Stender K, Bürger V, Nil R. Efficacy and tolerability of escitalopram in 12- and 24-week treatment of social anxiety disorder: randomized, double-blind, placebo-controlled, fixed-dose study. Depress Anxiety. 2004;19(4):241-248.

13. Nakasa H, Ariyoshi N, Kitada M. 1. [Drug metabolizing enzymes, e. CYP genotypes and expression regulation]. In: Sawada Y, editor. Genotypes and Pharmacokinetics/Effects - New Developments in the 21st Century in Tailor-made Drug Therapy. 1st ed. Osaka: Iyaku (Medicine and Drug) Journal Co, Ltd; 2001:158-171. Japanese.

14. Sato H, Hirayasu Y. [Escitalopram clinical pharmacokinetic study Single dose and multiple dose administration (Japan)]. Jpn J Clin Psychopharmacol. 2011;14(5):839-855. Japanese.

15. American Psychiatric Association. Diagnostic and Statistical Manual of Mental Disorders, Text Revision: DSM-IV-TR ${ }^{\mathrm{TM}}$. 4th ed. Washington, DC: American Psychiatric Association; 2000.

16. Otsubo T, Miyaoka H, Kamijima K, editors. [M.I.N.I. Brief Diagnostic Structured Interview for Mental Disease]. Tokyo: Seiwa Shoten Publishers; 2000. Japanese.

17. Asakura S, Inoue S, Sasaki F, et al. [Reliability and validity of the Japanese version of the Liebowitz Social Anxiety Scale]. Seishin Igaku (Clinical Psychiatry). 2002;44(10):1077-1084. Japanese. 
18. Montgomery SA, Asberg M. A new depression scale designed to be sensitive to change. Br J Psychiatry. 1979;134:382-389.

19. Posner K, Brown GK, Stanley B, et al. The Columbia-Suicide Severity Rating Scale: initial validity and internal consistency findings from three multisite studies with adolescents and adults. Am J Psychiatry. 2011; 168(12):1266-1277.
20. Yoshida T, Otsubo T, Tsuchida H, Wada Y, Kamijima K, Fukui K. [Reliability and validity of the Sheehan Disability Scale - Japanese version]. Jpn J Clin Psychopharmacol. 2004;7(10):1645-1653. Japanese.

21. Wade A, Despiegel N, Heldbo Reines E. Escitalopram in the long-term treatment of major depressive disorder. Ann Clin Psychiatry. 2006; 18(2):83-89.

\section{Publish your work in this journal}

Neuropsychiatric Disease and Treatment is an international, peerreviewed journal of clinical therapeutics and pharmacology focusing on concise rapid reporting of clinical or pre-clinical studies on a range of neuropsychiatric and neurological disorders. This journal is indexed on PubMed Central, the 'PsycINFO' database and CAS, and is the official journal of The International Neuropsychiatric Association (INA). The manuscript management system is completely online and includes a very quick and fair peer-review system, which is all easy to use. Visit http://www.dovepress.com/testimonials.php to read real quotes from published authors.

Submit your manuscript here: http://www.dovepress.com/neuropsychiatric-disease-and-treatment-journal 